\title{
Mixed integer quadratically-constrained programming model to solve the irregular strip packing problem with continuous rotations
}

\author{
Luiz H. Cherri ${ }^{1,3}$. Adriana C. Cherri ${ }^{2}$. \\ Edilaine M. Soler ${ }^{2}$
}

Received: 25 April 2017 / Accepted: 6 March 2018 / Published online: 14 March 2018

(C) Springer Science+Business Media, LLC, part of Springer Nature 2018

\begin{abstract}
The irregular strip packing problem consists of cutting a set of convex and nonconvex two-dimensional polygonal pieces from a board with a fixed height and infinite length. Owing to the importance of this problem, a large number of mathematical models and solution methods have been proposed. However, only few papers consider that the pieces can be rotated at any angle in order to reduce the board length used. Furthermore, the solution methods proposed in the literature are mostly heuristic. This paper proposes a novel mixed integer quadratically-constrained programming model for the irregular strip packing problem considering continuous rotations for the pieces. In the model, the pieces are allocated on the board using a reference point and its allocation is given by the translation and rotation of the pieces. To reduce the number of symmetric solutions for the model, sets of symmetry-breaking constraints are proposed. Computational experiments were performed on the model with and without symmetry-breaking constraints, showing that symmetry elimination improves the quality of solutions found by the solution methods. Tests were performed with instances from the literature. For two instances, it was possible to compare the solutions with a previous model from the literature and show that the proposed model is able to obtain numerically accurate solutions in competitive computational times.
\end{abstract}

Keywords Mixed integer non-linear programming - Nesting problems · Irregular pieces · Continuous rotations

Luiz H. Cherri

lhcherri@icmc.usp.br

1 University of São Paulo, Av. Trabalhador São-carlense, 400, São Carlos, SP 13566-590, Brazil

2 Universidade Estadual Paulista, Av. Eng. Luiz Edmundo Carrijo Coube, 14-01, Bauru, SP 17033-360, Brazil

3 Optimized Decision Making (ODM), Rua Alfredo Lopes 1717, Sala E10, São Carlos, SP 13560-460, Brazil 


\section{Introduction}

Irregular strip packing problems belong to a class of cutting problems and arise in many industries such as garment manufacturing, metal mechanic, furniture, footwear, among others. In a brief description, the problem consists of determining an efficient configuration of cutting a set of two-dimensional irregular polygonal pieces from a board with a fixed height and length long enough to be considered infinite. The objective is usually to minimize the length of the board used to cut all the pieces. Since the shape of pieces in these problems are irregular, an additional difficulty is to formulate the geometry of these problems, taking into account that it is necessary to define when two pieces allocated in a board are overlapping, touching or are separated. Furthermore, it should be ensured that the pieces are completely allocated on the board.

Irregular strip packing problems have been widely studied in the literature and belong to the class of NP-hard problems [10]. There are few studies in the literature that have used exact solution methods for irregular strip packing problems without any piece rotations. The first model was proposed by Carravilla et al. [5] in which the authors presented a solution method to solve the nesting problems based on constraint programming. In their model, the pieces can only be placed over discrete positions on the board. Also using a finite set of positions to place pieces on a board, Toledo et al. [22] proposed a mixed-integer model. Although the models proposed by Carravilla et al. [5] and Toledo et al. [22] were solved by exact methods, the solutions they obtained are subject to discretization points and will hardly ever be truly optimal.

Models where the pieces can be placed continuously on (X,Y)-coordinates of the board were proposed by Fischetti and Luzzi [9], Alvarez-Valdes et al. [1] and Cherri et al. [7]. Alvarez-Valdes et al. [1] redefined some structures of Fischetti and Luzzi's [9] model and proposed a branch and bound algorithm for their model. Attempting to combine the performance of discrete models and the accuracy of the linear models, Leão et al. [17] proposed a semi-continuous model, where the pieces can be placed on continuous positions on the $\mathrm{x}$-axis and on discrete positions on the y-axis. Cherri et al. [7] proposed a model that enable pieces to be rotated at a finite number of angles. The authors proposed the first mixed integer linear programming model that does not use the no-fit polygon to prevent overlapping between pieces.

Bennell et al. [2] considers a container (rectangle, circle, convex polygon) of variable sizes and only two irregular pieces bounded by circular arcs and/or line segments that can be continuously translated and rotated. Although this is not a strip packing problem, the authors proposed a mathematical model to represent the problem, taking into account allowable distances between pieces and between each piece and the edge of the container. The model uses the concept of phi-functions and provides new benchmark instances to find the containing region that has either a minimal area, perimeter or a homothetic coefficient of a given container.

An important characteristic observed in real world applications, especially in the leather and metal industry, is the possibility of rotating the pieces at any angle. Due to the fact that in these industries the orientation of the piece on the board is irrelevant, it is possible to make better use of the raw material when the pieces can be continuously rotated. Some papers from the literature have proposed heuristic procedures to solve this problem. Aiming to minimize waste, Fujita et al. [11] proposed a hybrid approach combining a genetic algorithm with a local minimization algorithm to solve nesting problems with convex polygons and a continuous 
rotation of the pieces. A computational example showed the effectiveness and potential of the approach.

Rocha et al. [19] represented the pieces by sets of overlapping circles, aiming to achieve a certain degree of approximation while minimizing the necessary number of circles, allowing for any possible rotation. Rocha et al. [20] proposed a two-phase approach to the nesting problem with continuous rotations based on a common compaction strategy which relies on the observable concept that the core structure of a layout is usually defined by the position and orientation of its largest pieces. Liao et al. [18] proposed an algorithm to allocate irregular pieces in a rectangular sheet without overlapping based on the rubber band packing algorithm. This algorithm simulates the physical movements of a set of pieces wrapped by a rubber band. The main advantage of the proposed heuristic is its ability to translate, slide and rotate the pieces into a compact layout obtaining a good solution in an acceptable time.

Exact solutions methods for solving mathematical models for irregular strip packing problems with continuous rotations were proposed by some authors. Kallrath [16] developed mathematical models to describe the problem of cutting circles, rectangles and convex polygons while minimizing the area of the rectangular board used. An exact algorithm based on the idea of the separating hyper-plane theorem to ensure that the pieces do not overlap with each other was proposed. Cases including a maximum of 10 circles or circles and rectangles were solved in a short time to global optimality. According to the author, in tests with instances involving non-rectangular polygons, it is difficult to obtain small gaps.

The mathematical model proposed by Chernov et al. [6] also considered irregular pieces with continuous rotations. Piece overlap was evaluated evaluated using phi-functions. Although the authors proved the exactness of the proposed model, a heuristic that uses a variation of the proposed model was developed. Their heuristic method found good quality solutions in an acceptable computational time, however their computational results did not evaluate how far these solutions were from optimality.

In Jones [15], an exact algorithm was developed allowing the pieces to be freely translated or rotated. To solve the problem, circles are inscribed inside of each irregular piece and then the non-overlap constraints are formulated ensuring that the circles inscribed in one piece do not overlap the inscribed circles from another piece. The problem was formulated as a quadratic programming problem and it was solved to optimality by non-linear global solvers. Although the strategy used by the author is interesting, the pieces may not be represented accurately, affecting the evaluation of overlapping between the pieces.

Stoyan et al. [21] formulated a basic irregular placement problem for cutting and packing problems. The authors considered the pieces bounded by circular arcs and line segments and allowed continuous rotations and translations within the rectangular and circular containers. An exact non-linear programming model for the problem using ready-to-use phi-functions was proposed. The solution presented by the proposed algorithm improved the best published results for some benchmark instances provided that free piece rotations are allowed.

Although some mathematical formulations and solution methods for the irregular strip packing problem with continuous piece rotations were proposed by some researchers, in most of these studies heuristic methods were used to solve the problem. Furthermore, the exact solution methods proposed have some limitations in terms of representing the pieces and high computational time.

In this paper, a mathematical formulation for the irregular strip packing problem with continuous rotations of the pieces is proposed and solved by exact methods proposed in the solvers COUENNE, SCIP and BARON. This formulation has linear and quadratic constraints and differs from the Kallrath's [16] model in the way the non-overlap constraints are designed. Furthermore, the proposed model can handle non-convex pieces. 
The main contributions of this paper are: (1) a mixed integer quadratically constrained model is proposed to represent the irregular strip packing problem using pieces with continuous rotations; (2) the model invokes only simple geometric structures to be built making them easy to be reproduced; (3) great precision when packing polygonal pieces; and (4) smaller or equal computational times when compared to the only two the instances solved by another exact method from the literature and solution for other challenging instances of the literature.

After this introductory section, Sect. 2 presents the irregular strip packing problem and some definitions used throughout the paper. The mixed integer quadratically-constrained model for the irregular strip packing problem is proposed in Sect. 3. Section 4 discusses the bounds for this problem and how they can be obtained, as well as a set of symmetrybreaking constraints and domain reductions which can be used in the model without affecting its optimality. The computational experiments performed with the proposed mathematical models are depicted in Sect. 5. Finally, some conclusions are drawn in Sect. 6.

\section{Definitions and basic structures}

In the irregular strip packing problem addressed in this paper, a set of $N$ convex and nonconvex polygonal pieces must be placed on a board with a fixed height $H$ and an infinite length. These pieces can be rotated at any angle, they must be completely inside the board and must not overlap. The objective is to minimize the board length used to cut all the pieces.

To mathematically formulate this problem, the polygonal pieces are represented by a set of points ordered in a counterclockwise direction. A reference point is used to indicate the locus of the piece on the board, i.e., this point is used to translate the piece into the board and to rotate the piece around it. Figure 1 illustrates a piece, its reference point, and the coordinates of the system.

Note that the reference point can be arbitrarily chosen, however, in this study, we consider the leftmost vertex of the piece as the reference point. In case of a tie, the bottommost vertex is chosen among the candidates.

A decomposition of the non-convex pieces into convex pieces is used to formulate the mathematical model. For this, Greene's dynamic programming algorithm for optimal partitioning [14] is used. By this algorithm, the pieces are decomposed in a minimum number of convex parts, considering that new vertices cannot be included into the polygon. Although this decomposition is not unique, using the algorithm the same decomposition will always be found. Figure 2 illustrates this decomposition for the piece given in Fig. 1. Note that all the vertices of the decomposed piece (Fig. 2) exist on the complete piece (Fig. 1).

Fig. 1 Representation of a piece and its reference point

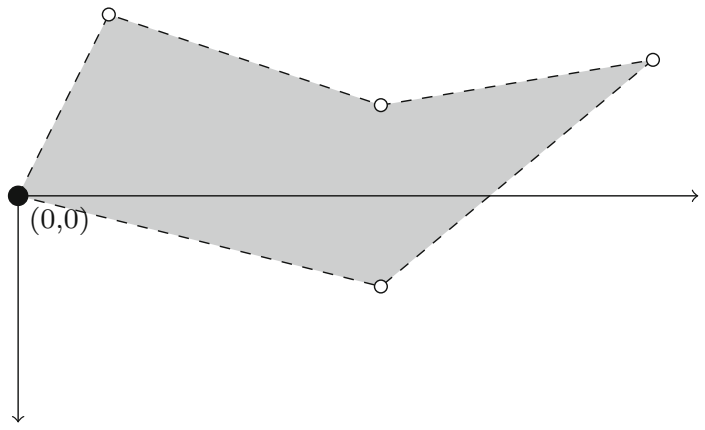


Fig. 2 Convex decomposition of the piece presented in Fig. 1

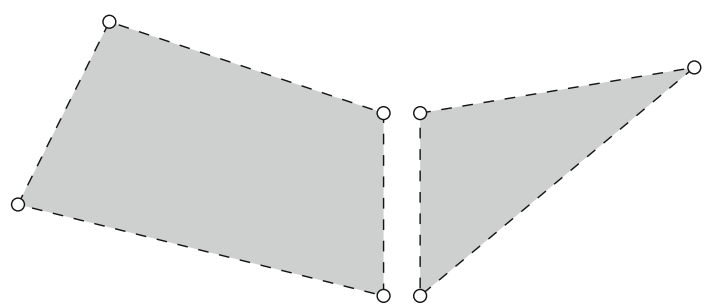

Specifically, let $K_{i}$ be the set of vertices of piece $i, i=1, \ldots, N$, and $K_{i, s}$ the set of vertices of its $s$-th decomposed part, $s=1, \ldots, S_{i}$, where $S_{i}$ is the number of convex parts of piece $i$. Thus, $K_{i, s} \subset K_{i}$ and $\bigcup_{s=1}^{S_{i}} K_{i, s} \equiv K_{i}$. These two piece representations are used in this paper to illustrate the relation between pieces by constraints.

Another important information is the relation between a point and a line. To obtain this value, the D-function [3] is used. Precisely, given a oriented line and a point, the D-function value identifies the relative position of the point and the line. This equation is derived from the line point distance equation. Consider two points $A\left(a_{x}, a_{y}\right)$ and $B\left(b_{x}, b_{y}\right)$ from a line and a point $P\left(p_{x}, p_{y}\right)$. The normal vector of a line $\overrightarrow{A B}$ is given by $\vec{v}=\left(a_{y}-b_{y}, b_{x}-a_{x}\right)$. Thus, the distance from point $P$ to line $\overrightarrow{A B}$ is obtained by Formula (1).

$$
\frac{\left|\left(a_{x}-b_{x}\right)\left(a_{y}-p_{y}\right)-\left(a_{y}-b_{y}\right)\left(a_{x}-p_{x}\right)\right|}{\sqrt{\left(a_{x}-b_{x}\right)^{2}+\left(a_{y}-b_{y}\right)^{2}}},
$$

where |.| is the absolute value of the equation.

Considering (1), only positive values are obtained, which is correct as they represent an Euclidean distance. However, the sign of the distance between the point and the line is an important piece of information for our proposal. Moreover, by removing the denominator of Eq. (1), the relative position between a point and a line can be identified. The D-function of line $\overrightarrow{A B}$ and point $P$ is given by Eq. (2).

$$
D_{A B P}=\left(a_{x}-b_{x}\right)\left(a_{y}-p_{y}\right)-\left(a_{y}-b_{y}\right)\left(a_{x}-p_{x}\right) .
$$

The D-function has a simpler formula compared with the real distance equation and provides the information of the relative position between a point and a line. If $D_{A B P}$ is equal to zero, point $P$ is over line $\overrightarrow{A B}$; if $D_{A B P}$ is lower than zero, point $P$ is to the right of line $\overrightarrow{A B}$; and if $D_{A B P}$ is greater than zero, point $P$ is to the left of line $\overrightarrow{A B}$.

\section{Translate and Rotate Piece Model (TRPM)}

Throughout this section, the constraints for the Translate and Rotate Piece Model (TRPM) are described. The most difficult and important constraints ensure that the pieces are completely inside the board and they do not overlap.

To formulate the constraints, consider that the locus of piece $i$ on the board is defined by a translation of its reference point $\left(x_{i}, y_{i}\right)$ and a rotation $\theta_{i}$ of the piece around its reference point. A single angle $\theta_{i}$ is sufficient to define the rotation matrix for piece $i$, but as the sine and cosine functions are non-convex, the computational effort to calculate the sine and cosine for each angle in the constraints might unnecessarily increase the complexity of the model, affecting its resolution. Therefore, consider the variables $s_{i}$ and $c_{i}$, as well as Eq. (3). 


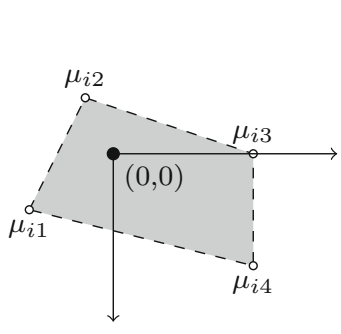

(a)
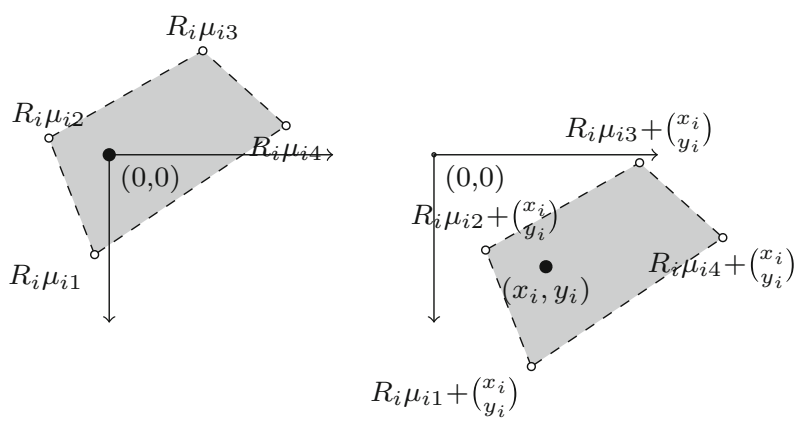

(c)

Fig. 3 Placing the piece on the board. a Piece before it is rotated or translated, b rotated piece and $\mathbf{c}$ piece translated and rotated

$$
s_{i}^{2}+c_{i}^{2}=1, \quad i=1, \ldots, N .
$$

Considering Eq. (3), the values of $s_{i}$ and $c_{i}$ are exactly the same value as $\sin \left(\theta_{i}\right)$ and $\cos \left(\theta_{i}\right)$, thus the $R_{i}=\left[\begin{array}{cc}c_{i} & s_{i} \\ -s_{i} & c_{i}\end{array}\right]$ represents the two dimensional rotation matrix. Consider $\mu_{i l, x}\left(\mu_{i l, y}\right)$ as the signed distance between vertex $l$ of piece $i$ and $x_{i}\left(y_{i}\right)$ as illustrated in Fig. 3a, where $\mu_{i l}=\left(\mu_{i l, x}, \mu_{i l, y}\right)^{T}$. The position of each vertex of the piece after a rotation around the reference point is illustrated in Fig. 3b. Figure $3 \mathrm{c}$ illustrates the positions of each vertex of the piece after rotation and translation to the desired point.

The position of vertex $l$ of each piece $i\left(p_{i l}\right)$ is formally defined by Eqs. (4) and (5).

$$
\begin{aligned}
& p_{i l, x}=x_{i}+\mu_{i l, x} c_{i}+\mu_{i l, y} s_{i}, \quad i=1, \ldots, N, l \in K_{i}, \\
& p_{i l, y}=y_{i}+\mu_{i l, y} c_{i}-\mu_{i l, x} s_{i}, \quad i=1, \ldots, N, l \in K_{i} .
\end{aligned}
$$

As in the irregular strip packing problem the board has a fixed height $(H)$, one can ensure that the pieces are entirely inside the board by maintaining the piece points inside the board, as expressed by constraints (6) and (7).

$$
\begin{aligned}
& p_{i l, x} \geq 0, \quad i=1, \ldots, N, l \in K_{i}, \\
& 0 \leq p_{i l, y} \leq H, \quad i=1, \ldots, N, l \in K_{i} .
\end{aligned}
$$

Note that only one bound was imposed for $p_{i l, x}$ as the board length is considered as an open dimension in the problem.

To ensure that the pieces do not overlap, the D-function presented in Sect. 2 is used. Let $l$ be a vertex of part $s$ of piece $i$ and $\bar{l}$ its consecutive vertex. Consider also vertex $m$ of part $s^{\prime}$ of piece $j$. If inequality (8) holds for all $m \in K_{j, s^{\prime}}$, it ensures that part $s^{\prime}$ of piece $j$ does not overlap part $s$ of piece $i$ by being on the left side of the line defined by vertices $l$ and $\bar{l}$. Figure 4 illustrates a case where inequality (8) holds.

$$
\left(p_{i l, x}-p_{i \bar{l}, x}\right)\left(p_{i l, y}-p_{j m, y}\right)-\left(p_{i l, y}-p_{i \bar{l}, y}\right)\left(p_{i l, x}-p_{j m, x}\right) \leq 0
$$

According to the Separating Axis Theorem, it can be stated that in a feasible solution, for each pair of parts of different pieces, there is one line which passes by an edge of one of the parts that ensures these piece parts are separated into different sides of the line. Note that this line could pass on an edge of one of both of the parts of the pieces. Further details about the Separating Axis Theorem can be found in Gottschalk [13]. 

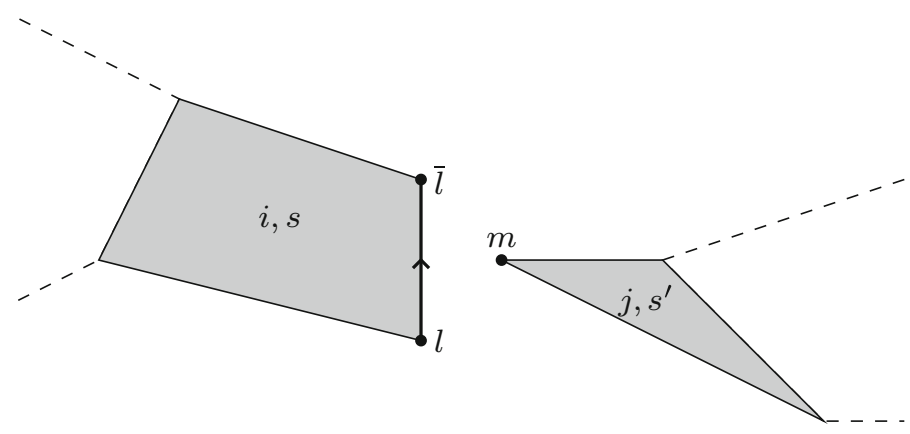

Fig. 4 Example of a case in which Eq. (8) holds

To simultaneously consider all these constraints, consider variable $\gamma_{j s^{\prime}}^{i s l}$, which is 1 if part $s^{\prime}$ of polygon $j$ is to the left side of the line that pass by vertices $l$ and $\bar{l}$ of part $s$ of polygon $i$, and 0 otherwise. Thus, two parts of the different pieces are separated if constraint ( 9 ) holds for one or more $\gamma_{j s^{\prime}}^{i s l}$ equal to one.

$$
\begin{gathered}
\left(p_{i l, x}-p_{i \bar{l}, x}\right)\left(p_{i l, y}-p_{j m, y}\right)-\left(p_{i l, y}-p_{i \bar{l}, y}\right)\left(p_{i l, x}-p_{j m, x}\right) \leq\left(1-\gamma_{j s^{\prime}}^{i s l}\right) M, \\
i, j=1, \ldots, N, i \neq j, s=1, \ldots, S_{i}, s^{\prime}=1, \ldots, S_{j}, l, \bar{l} \in K_{i, s}, m \in K_{j, s^{\prime}}
\end{gathered}
$$

where $M$ is a number large enough to relax constraint (9) when $\gamma_{j s^{\prime}}^{i s l}$ is equal to zero. The value of this constant will be analyzed in Sect. 4.2.

If, for a given part $s$ of piece $i$ and part $s^{\prime}$ of piece $j$, exactly one variable $\gamma_{j s^{\prime}}^{i s l}$ is equal to one (activated), then these parts do not overlap as formulated by constraints (10).

$$
\begin{aligned}
\sum_{l \in K_{i, s}} \gamma_{j s^{\prime}}^{i s l}+\sum_{m \in K_{j, s^{\prime}}} \gamma_{i s}^{j s^{\prime} m}=1, \quad & i, j=1, \ldots, N, i \leq j, \\
s & =1, \ldots, S_{i}, s^{\prime}=1, \ldots, S_{j} .
\end{aligned}
$$

Although a set of $\gamma_{j s^{\prime}}^{i s l}$ variables can be activated to avoid the overlap between these parts, selecting only one variable to activate eliminates symmetries without losing the solution optimality. Figure 5 illustrates the symmetry elimination. In the figure, although variables $\gamma_{j s^{\prime}}^{i s l}$ and $\gamma_{j s^{\prime}}^{i s l^{\prime}}$ activate different regions to place part $s^{\prime}$ of piece $j$, there is an intersection area among them. With constraint (10), a vertex of part $s^{\prime}$ of piece $j$ can be placed on this intersection area by either $\gamma_{j s^{\prime}}^{i s l}=1$ or $\gamma_{j s^{\prime}}^{i s l^{\prime}}=1$, however, both variables cannot be one at the same time. This does not eliminate feasible solutions, as the intersection region can still be used to place the vertices from part $s^{\prime}$ of piece $j$, This restriction also helps the solution methods to reduce the number of solutions to be explored until the optimality is proved.

The objective the problem is to minimize the length of the board $(L)$ used to cut of all the pieces. Constraint (11) supports this objective:

$$
p_{i l, x} \leq L, \quad i=1, \ldots, N, l \in K_{i} .
$$

The complete Translate and Rotate Piece Model (TRPM) is presented in the following. It is a mixed integer quadratically-constrained programming model.

min: $L$

$$
\text { s. t.: } 0 \leq p_{i l, x} \leq L, \quad i=1, \ldots, N, l \in K_{i},
$$


Fig. 5 Example of symmetry elimination

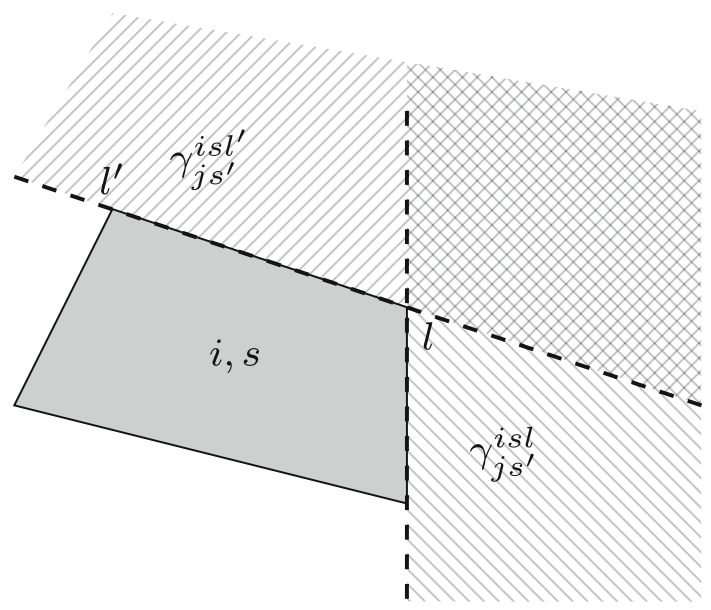

$$
\begin{aligned}
& 0 \leq p_{i l, y} \leq H \\
& s_{i}^{2}+c_{i}^{2}=1 \\
& p_{i l, x}=x_{i}+\mu_{i l, x} c_{i}+\mu_{i l, y} s_{i}, \\
& p_{i l, y}=y_{i}+\mu_{i l, y} c_{i}-\mu_{i l, x} s_{i}, \\
& \left(p_{i l, x}-p_{i \bar{l}, x}\right)\left(p_{i l, y}-p_{j m, y}\right)- \\
& \left(p_{i l, y}-p_{i \bar{l}, y}\right)\left(p_{i l, x}-p_{j m, x}\right) \leq\left(1-\gamma_{j s^{\prime}}^{i s l}\right) M,
\end{aligned}
$$$$
i=1, \ldots, N, l \in K_{i},
$$$$
i=1, \ldots, N \text {, }
$$$$
i=1, \ldots, N, l \in K_{i},
$$$$
i=1, \ldots, N, l \in K_{i} \text {. }
$$$$
i, j=1, \ldots, N, i \neq j,
$$$$
s=1, \ldots, S_{i}, l, \bar{l} \in K_{i, s},
$$$$
s^{\prime}=1, \ldots, S_{j}, m \in K_{j, s^{\prime}},
$$$$
\sum_{l \in K_{i, s}} \gamma_{j s^{\prime}}^{i s l}+\sum_{m \in K_{j, s^{\prime}}} \gamma_{i s}^{j s^{\prime} m}=1
$$$$
i, j=1, \ldots, N, i \leq j,
$$

$s_{i}, c_{i} \in[-1,1]$,

$$
\begin{array}{r}
s=1, \ldots, S_{i}, s^{\prime}=1, \ldots, S_{j}, \\
i=1, \ldots, N, \\
i=1, \ldots, N, l \in P_{i, u}, \\
i=1, \ldots, N, \\
i, j=1, \ldots, N, u=1, \ldots, S_{i}, \\
v=1, \ldots, S_{j}, l \in P_{i, u},
\end{array}
$$

$L \in \mathbb{R}$

\section{Bounds, estimations and symmetries}

In Sect. 3, a mixed integer quadratically-constrained model was proposed to represent the irregular strip packing problem with continuous rotations. The model contains many symmetric solutions and poor bounds due to the big- $M$ term in the constraints (9). This section is dedicated to studies better bounds, tighter $M$ terms and some symmetry-breaking constraints that can help speed up the solution methods. 


\subsection{Bounds}

Finding good lower bounds for irregular cutting and packing problems is a difficult task that worsens when rotations are considered. The most common and simple estimation is considered where pieces can fit perfectly inside the board, that is, the lower bound is obtained by the sum of the piece's areas divided by the board height. Other bounds can be obtained, however, in many cases the problem to be solved might be as difficult as it is to solve the complete problem. For example, using Jones' [15] model with a restricted number of circles to represent the pieces leads to a lower bound for the problem, however, the computation time to obtain this lower bound might be prohibitive.

Although studying good lower bounds encourages researchers to reduce the gaps from the literature, in most cases when they are imposed in the model they can affect the resolution as they lie about the nodes current bounds. Thus, the lower bounds will not be used in the resolution of the proposed model.

Upper bounds for the irregular strip packing problem can be found by heuristic methods. Good upper bounds can improve the convergence speed of the methods by not exploring parts of the solution space in which only worse solutions could be found. In cases where the pieces can be rotated in a finite set of angles, many heuristics have been proposed $[8,12]$. These solutions can be used as an upper bound for the irregular strip packing problem with continuous rotations. Furthermore, many studies heuristically address the irregular strip packing problem considering continuous piece rotations to find good upper bounds $[18,20]$.

A simple way to obtain upper bounds for the problem is to solve one of the proposed models by a mixed integer non-linear programming solver which searches for the local optimal, thus finding a local solution to the problem.

\subsection{The big- $M$ term value}

It is important to analyze how big the $M$ term used in the non-overlap constraints must be to ensure that the model finds optimal solutions. Although a huge value is sufficient to completely disable these constraints, it is known that a tighter $M$ can boost the permanence of solution methods by improving the quality of the model relaxations.

Consider the non-overlap constraints (9). Estimating $M$ is only necessary if $\gamma_{j s^{\prime}}^{i s l}$ is equal to 0 , thus:

$$
M_{i j l} \geq\left(p_{i l, x}-p_{i \bar{l}, x}\right)\left(p_{i l, y}-p_{j m, y}\right)-\left(p_{i l, x}-p_{j m, x}\right)\left(p_{i l, y}-p_{i \bar{l}, y}\right)
$$

Consequently, by absolute value property it is known that inequality (25) holds.

$$
\begin{aligned}
& \left(p_{i l, x}-p_{i \bar{l}, x}\right)\left(p_{i l, y}-p_{j m, y}\right)-\left(p_{i l, x}-p_{j m, x}\right)\left(p_{i l, y}-p_{i \bar{l}, y}\right) \\
& \quad \leq\left|p_{i l, x}-p_{i \bar{l}, x}\right|\left|p_{i \bar{l}, y}-p_{j m, y}\right|+\left|p_{i \bar{l}, x}-p_{j m, x}\right|\left|p_{i l, y}-p_{i \bar{l}, y}\right|
\end{aligned}
$$

The values of $\left|p_{i l, x}-p_{i \bar{l}, x}\right|$ and $\left|p_{i l, y}-p_{i \bar{l}, y}\right|$ are, respectively, the horizontal and vertical distances for two consecutive vertices of polygon $i$. These distances could not be larger than the euclidean distance $\left(d_{i l \bar{l}}\right)$ between $\left(p_{i l, x}, p_{i l, y}\right)$ and $\left(p_{i \bar{l}, x}, p_{i \bar{l}, y}\right)$. In addition, the values of $\left|p_{i \bar{l}, y}-p_{j m, y}\right|$ and $\left|p_{i \bar{l}, x}-p_{j m, x}\right|$ are respectively bounded by the $H$ (board height) and $L$ (board length). Thus, the following inequality holds,

$$
\left|p_{i l, x}-p_{i \bar{l}, x}\right|\left|p_{i \bar{l}, y}-p_{j m, y}\right|+\left|p_{i \bar{l}, x}-p_{j m, x}\right|\left|p_{i l, y}-p_{i \bar{l}, y}\right| \leq d_{i l \bar{l}}(H+L)
$$


Then, the $M$ term can be defined as in Eq. (27).

$$
M_{i l \bar{l}}=d_{i l \bar{l}}(H+L)
$$

Using the therm defined in (27) in inequality (9) causes a product of $L$ and $\gamma_{j s^{\prime}}^{i s}$. For some solution methods, it can hamper the resolution, therefore, an alternative is use an upper bound for the board length $(\bar{L})$ instead of using variable $L$, turning $M$ into a constant.

\subsection{Symmetry-breaking constraints}

Symmetric pieces can lead to a set of redundant solutions for the problem. Precisely, if a given piece $i$ is a regular polygon, variables $s_{i}$ and $c_{i}$ can have reduced domains. For example, if a square must be packed, it is not necessary to evaluate all the rotations in the range $[0,2 \pi]$. As a matter of fact, evaluating only the angles in the range $[0, \pi / 2]$ is sufficient to maintain the solution optimality.

To generalize this idea, consider $\phi_{i}$, the symmetry angle which represents the smallest angle whereby, if piece $i$ is rotated, it remains equal to piece $i$ at rotation 0 . Thus, the rotation angle of a symmetric piece $i$ can be reduced to the range $\left[0, \phi_{i}\right]$. This range can also be rewritten as $\left[-\phi_{i} / 2, \phi_{i} / 2\right]$ and be used to redefine the domain of $c_{i}$ variables. Specifically, given a symmetric piece $i$, the domains of variables $c_{i}$ are reduced to $\left[\cos \left(\phi_{i} / 2\right), 1\right]$. Note that to reduce the domains of the variables $c_{i}$ is sufficient to restrict the rotation angle into the desired interval and no changes in the domain of variable $s_{i}$ are needed.

For each solution found by the model, there is a symmetric one which can be obtained by rotating the solution by $\pi$. If one or more ordering subset constraints were introduced into the problem, then this type of symmetry is already eliminated. However, if no ordering subset constraint is imposed, it may be assumed that one of the piece vertices can be allocated only on one half of the board, thereby eliminating symmetric solutions. Specifically, the domain of the $y$ coordinate of the reference point of piece $i$ is reduced to the interval $\left[0, \frac{H}{2}\right]$.

Another type of redundant solution emerges when pieces with the same shape must be packed. Precisely, if pieces $i$ and $j$ have the same shape, then symmetries can be eliminated ensuring that the reference point of piece $i$ precedes to the reference point of piece $j$ in a horizontal direction. Therefore, a subset of ordering constraints is introduced, that is, $x_{i} \leq x_{j}$, if $i<j$ and pieces $i$ and $j$ have the same shape.

\section{Computational results}

In this section, the performance of the proposed model when solved by three different global optimization solvers is compared. Furthermore, the results found in the proposed formulation are compared with the state-of-art model from the literature.

The computational experiments were conducted using a computer with an Intel i7 4790 processor using 16 gigabytes of memory and Ubuntu 16.04 operating system. The models were implemented using the General Algebraic Modeling System. All the tests were carried out using GAMS 24.9 [4]. The time limit adopted in the experiments was $7 \mathrm{~h}(25,200 \mathrm{~s})$ which is the same used in Jones [15]. Three global optimization solvers were used to solve the models: COUENNE 0.5, SCIP 3.2 and BARON 16.5.16. These solvers were chosen because they are free for academics (actually COUENNE is distributed under the Common Public License).

For SCIP and BARON, the default solver options were used, however, some changes were made in COUENNE solver options. The linear and mixed integer linear solvers were 
changed form CLP/CBC (COIN-OR project) to CPLEX (IBM). COUENNE uses a nonlinear branch and bound to solve the problems. The default search strategy of the method is strong-branching which generally explores less nodes than other strategies. However, in preliminary computational experiments, the reliability branching showed a better performance than strong-branching in terms of computational time and solution quality. This improvement was expected as reliability branching is generally the best branching choice for mixed integer linear programming model solvers.

In Sects. 5.1 and 5.2, the TRPM model without and with symmetry-breaking constraints were respectively analyzed. The instances used in this phase were taken from Jones [15] and are called: four-part and six-part. In both instances, all the pieces have convex shapes and, in the optimal solution, the pieces fit perfectly on the board. The four-part instance has four pieces and the board height is equal to 10 and the six-part instance has six pieces and the board height is also equal to 10 .

Although these instances only deal with convex pieces, they are the only instances from the literature solved by an exact method.

To further analyze the performance of the proposed models, in Sect. 5.3 some instances with convex and non-convex pieces from the literature were used. In these instances, the pieces do not fit perfectly on the board and were taken and adapted from the ESICUP webpage (EURO Special Interest Group on Cutting and Packing). Specifically, six instances were used. Three and Shapes4 were used without any change. Blaze2[1] is a copy of each piece type of instance Blaze[2]. Poly1A[1-5], Poly1A[6-10] and Ploy1A[11-15] are derived from Poly1A instance and have a board of height 12 . The number in brackets represents the range of the piece types contained in them, i.e., Poly1A[1-5] contain the pieces 1 to 5 from Poly1A instance.

It is important to highlight that for some instances, the figure that represents the feasible solution gives the impression that they can easily be improved. However, this may not possible due to the various constraints for all pairs of pieces that limit the movements of each other, and the heuristics used by the solvers to find and improve the solutions.

\subsection{TRPM model without symmetry-breaking constraints}

In this section, the proposed TRPM model without the symmetry-breaking constraints is solved. Two variations of the model are analyzed: one using the $M$ term estimated with variable $L$ and the other with $M$ estimated with an upper bound of the board length $\bar{L}$. In this phase, the value of $\bar{L}$ is equal to 20 (two times the board height). These tests aim to verify if the complexity introduced having a slightly tighter $M$ benefits the quality and time of the obtained solutions.

Table 1 shows the results found for the four-part instance by three solvers. In the table, the first column shows the name of the solver used to solve the instance and the second column depicts the information in the line: Upper Bound (UB), Lower Bound (LB) and Computational Time (Time). The third column shows the results for the model with the big- $M$ estimation using the constant $\bar{L}\left(T R P M_{E}\right)$. The results for the model with the big- $M$ estimation with variable $L\left(T R P M_{L}\right)$ are shown in the fourth column.

Looking for the solvers, SCIP found better solutions compared with COUENNE and BARON for both models. In fact, SCIP found the optimal solution for TRPM $E$ in $48 \mathrm{~s}$ and for $T R P M_{L}$ in $64 \mathrm{~s}$ while the other formulations could not prove the solution optimality within the time limit imposed. 
Table 1 Results found solving four-part instance without symmetry breaking constraints

\begin{tabular}{lll}
\hline & TRPM $_{E}$ & TRPM $_{L}$ \\
\hline COUENNE & & \\
UB & 7.021 & 6.889 \\
LB & 1.725 & 1.844 \\
Time & TL & TL \\
SCIP & & \\
UB & 5.000 & 5.000 \\
LB & 5.000 & 5.000 \\
Time & $48^{\mathrm{a}}$ & $64^{\mathrm{a}}$ \\
BARON & & \\
UB & 5.000 & 6.000 \\
LB & 3.125 & 3.125 \\
Time & TL & TL \\
\hline
\end{tabular}

Fig. 6 a Presents the optimal solution for four-part instance with length 5. A feasible solution with length 6 is also presented in b

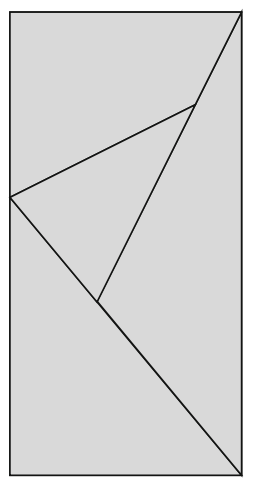

(a)

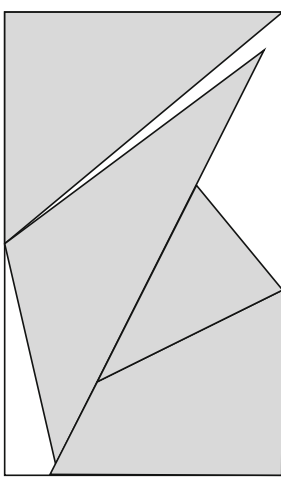

(b)

The $T R P M_{L}$ formulation had slightly better upper and lower bounds than $T R P M_{E}$ when COUENNE was chosen to solve it. Specifically, the first one had a GAP $(1-L B / U B)$ of $73.2 \%$ while the second had a GAP of $75.4 \%$.

Using BARON, the best upper and lower bounds were found using TRPM with estimated $M$. The GAP found by $T R P M_{E}$ was $37.5 \%$ while the GAP found by $T R P M_{L}$ was $44.2 \%$.

Figure 6 a illustrates the best solution for the problem found by SCIP. Note that as the pieces fit perfectly onto the board, there is no doubt about its optimality. The solution found using the TRPM $M_{L}$ in BARON is presented in Fig. $6 \mathrm{~b}$.

Table 2 shows the results for the six-part instance. This table has the same type of content as Table 1.

For the six-part instance, the best upper bound was found by COUENNE using the TRPME formulation and the best lower bound was SCIP in both formulations. COUENNE and BARON did not improve the lower bound within the time limit. SCIP found better lower bounds, however, it ran out of memory.

Comparing the formulations, for the six-part instance, $T R P M_{E}$ always found better upper bounds than $T R P M_{L}$ when using the same solver. 
Table 2 Results found solving six-part instance without symmetry breaking constraints

\begin{tabular}{lll}
\hline & TRPM $_{E}$ & TRPM $_{L}$ \\
\hline COUENNE & & \\
UB & 13.816 & 14.439 \\
LB & 0.000 & 0.000 \\
Time & TL & TL \\
SCIP & & \\
UB & 14.176 & 14.504 \\
LB & 4.491 & 4.491 \\
Time & $9891^{\mathrm{a}}$ & $4982^{\mathrm{a}}$ \\
BARON & & \\
UB & 14.314 & 15.096 \\
LB & 0.000 & 0.000 \\
Time & TL & TL \\
\hline
\end{tabular}

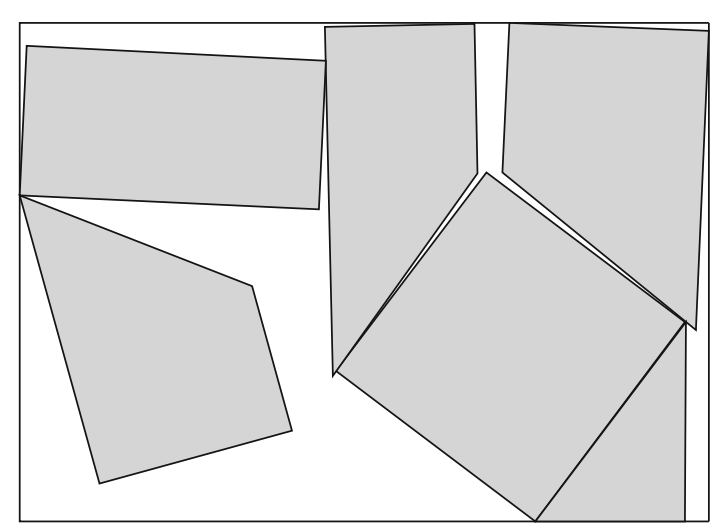

Fig. 7 Feasible solution for the six-part instance with length equal to 13.82
TL, time limit was reached $(25,200.00 \mathrm{~s})$

a Out of memory

Figure 7 illustrates the solution for the six-part instance found by $T R P M_{E}$ using COUENNE.

\subsection{TRPM model with symmetry-breaking constraints}

In this section, the upper bounds and symmetry-breaking constraints proposed in Sect. 4 are used to build $T R P M_{E}$ and $T R P M_{L}$ models. $T R P M_{E}$ and $T R P M_{L}$ with symmetry-breaking constraints are represented by $T R P M_{E}^{S B C}$ and $T R P M_{L}^{S B C}$, respectively. The initial solution (upper bound) used was the same given in Jones [15] and presented in Fig. 8. This local optimum is used as an initial solution in $T R P M_{E}^{S B C}$ and $T R P M_{L}^{S B C}$.

Table 3 presents the results for the four-part instance. The columns of this table present the same type of content as the columns in Table 1. It is important to highlight that COUENNE uses the initial solution only to guide the search and not as a feasible solution, thus the solutions found were worse than the initial solution.

The results found in Table 3 are better or equal to the ones found in Table 1 in terms of upper bounds, lower bounds and computational time.

In Table 3, except for the lower bounds found by COUENNE, TRPM ${ }_{E}^{S B C}$ obtained results with better or equal quality compared with $T R P M_{L}^{S B C}$. Analyzing the solvers, it is undeni- 


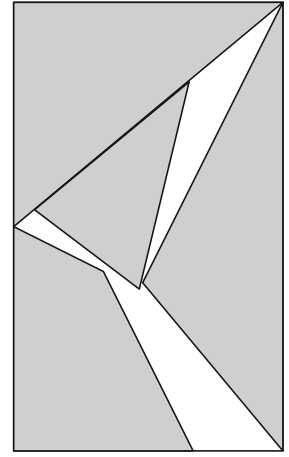

(a)

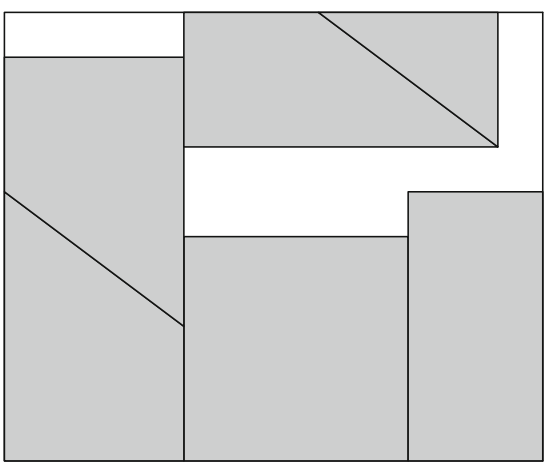

(b)

Fig. 8 a Initial solution for the four-part instance (length 6.0), b initial solution for the six-part instance (length 12.0)

Table 3 Results found solving the four-part instance with symmetry-breaking constraints

\begin{tabular}{lll}
\hline & TRPM $_{E}^{S B C}$ & TRPM $_{L}^{S B C}$ \\
\hline COUENNE & & \\
UB & 6.208 & 6.859 \\
LB & 1.926 & 2.081 \\
Time & TL & TL \\
SCIP & & \\
UB & 5.000 & 5.000 \\
LB & 5.000 & 5.000 \\
Time & $17^{\mathrm{a}}$ & $20^{\mathrm{a}}$ \\
BARON & & \\
UB & 5.000 & 6.000 \\
LB & 3.770 & 3.154 \\
Time & TL & TL \\
\hline
\end{tabular}

able that SCIP has better results with better quality than BARON and COUENNE in both formulations.

Despite the fact that COUENNE uses the initial solution only to guide the search, both upper and lower bounds were better when compared with the results in Table 1.

Jones [15] solved this instance considering 13 circles to represent the pieces in his most precise representation. The best solution found for the four-part instance was 5.000 in which the optimality was proved in 20,807 s considering the best approach.

Table 4 presents the results for the six-part instance. The columns of this table have the same type of content as the columns in Table 1. Despite the initial solution with value of 12.000 , COUENNE found a worse solution solving TRPM ${ }_{E}^{S B C}$ than solving $T R P M_{E}$. Furthermore, the initial upper bound was not improved by any method. However, all lower bounds in the table improved when compared with the results in Table 2, showing the importance of an initial solution and the symmetry-breaking constraints. The best lower bound was 7.903 and it was obtained by SCIP solver in $4491 \mathrm{~s}$. This value is better and was obtained in a shorter computational time than the lower bounds presented in Jones [15] for the same instance. 
Table 4 Results found solving the six-part instance in the models with symmetry-breaking constraints

$\begin{array}{llll} & \text { UB } & 14.453 & 14.132 \\ & \text { LB } & 4.419 & 4.419 \\ & \text { Time } & \text { TL } & \text { TL } \\ & \text { SCIP } & & \\ & \text { UB } & 12.000 & 12.000 \\ & \text { LB } & 7.903 & 6.677 \\ & \text { Time } & 4491^{\mathrm{a}} & 5790^{\mathrm{a}} \\ & \text { BARON } & & \\ & \text { UB } & 12.000 & 12.000 \\ & & 4.419 & 4.419 \\ \text { TL, Time limit was } & \text { reached } & \text { TL } & \text { TL } \\ \text { (25,200.00s) } & & \text { Time } & \text { TL }\end{array}$

Jones [15] improved the initial upper bound, however in his models the pieces are described by inner circles, admitting a small intersection among the pieces in some cases. As a consequence, near feasible solutions can be found and the optimal solution can only be proved adding more circles to the representation of pieces and solving the model again.

\subsection{TRPM model with convex and non convex pieces}

In this section, the proposed model is used to solve instances with convex and non-convex pieces which do not fit perfectly.

For each instance, the initial solution (upper bound) given to the formulations was found using BONMIN, a mixed integer non-linear programming solver proposed to find a local optimum. These solutions were found in less than $10 \mathrm{~min}$ and are presented in Fig. 9.

As in the previous sections, these instances were run in $T R P M_{E}^{S B C}$ and $T R P M_{L}^{S B C}$ models using three different solvers by $7 \mathrm{~h}$. The results obtained by the TRPM ${ }_{E}^{S B C}$ model are presented in Table 5 and the results obtained by $T R P M_{L}^{S B C}$ model are presented in Table 6 .

In these tables, the first column presents the instance name. For the COUENNE, SCIP and BARON solvers, the columns present respectively the lower bound (LB), upper bound (UB) and the computational time (TIME) in seconds to run the instances.

In Tables 5 and 6, the best lower bounds for all tested instances were found by SCIP. Moreover, except for Poly1A[1-5], SCIP found the best upper bound for all instances. For the instance Three, the three solvers found the optimal solution and among them, the SCIP solver for TRPM $M_{E}^{S B C}$ performed best, as the optimality of the solution was proved in $34 \mathrm{~s}$. For the SCIP solver in $T R P M_{E}^{S B C}$, the instances Poly1A[1-5], Poly1A[6-10] and Poly1A[11-15] did not reach the total run time due to the lack of computational resources. However, this solver had better upper bounds and lower bounds compared to COUENNE and BARON.

In both models, the SCIP solver improved the initial solution bounds for all the instances. BARON improved the initial solution bounds of three instances using each model. For the $T R P M_{E}^{S B C}$, the COUENNE solver improved the initial solution bound for three instances and found worse solutions for the other three instances. For the $T R P M_{L}^{S B C}$, COUENNE also improved the initial bounds for three instances, but it did not find a feasible solution for two instances and found a worst solution than the initial bound for one instance. 


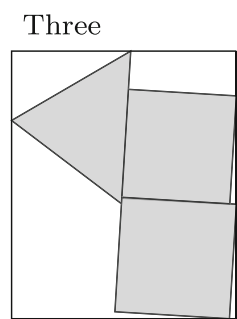

Length: 5.88

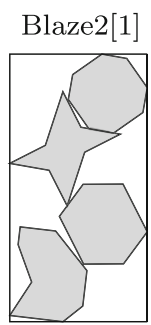

Length: 7.70

Shapes 4

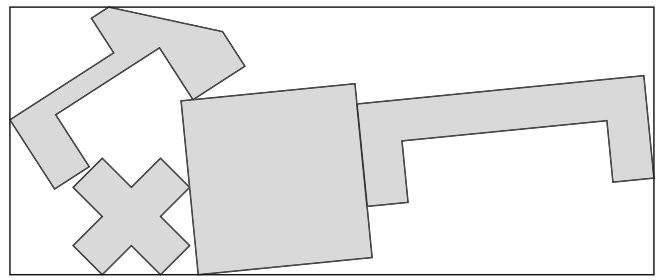

Length: 32.27

Poly1A[1-5]

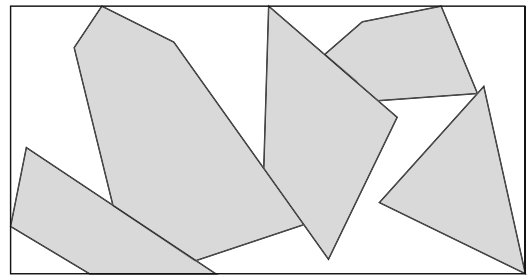

Length: 23.08

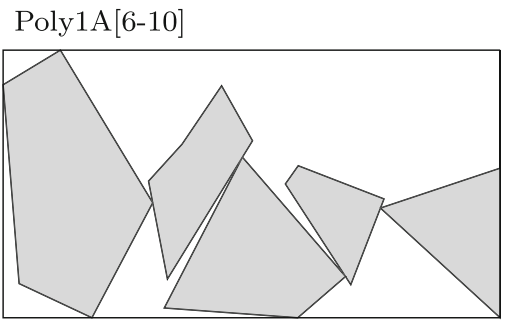

Length: 20.28

Poly1A[11-15]

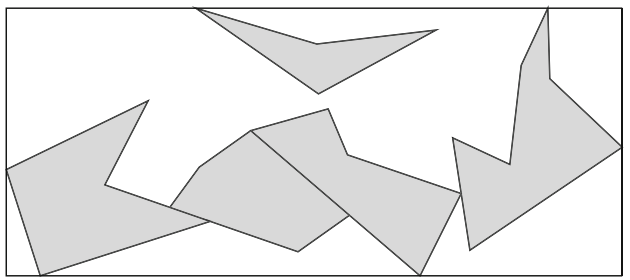

Length: 27.61

Fig. 9 Initial solutions

Table 5 Results found solving instances using $T R P M_{E}^{S B C}$ model

\begin{tabular}{|c|c|c|c|c|c|c|c|c|c|}
\hline \multirow[t]{2}{*}{ Instance } & \multicolumn{3}{|c|}{ COUENNE } & \multicolumn{3}{|l|}{ SCIP } & \multicolumn{3}{|c|}{ BARON } \\
\hline & LB & UB & TIME & LB & UB & TIME & LB & UB & TIME \\
\hline Three & 5.495 & 5.495 & $354^{\mathrm{b}}$ & 5.495 & 5.495 & $34^{\mathrm{b}}$ & 5.495 & 5.495 & $418^{\mathrm{b}}$ \\
\hline Blaze2 [1] & 2.828 & 10.297 & $\mathrm{TL}$ & 4.000 & 7.540 & $\mathrm{TL}$ & 2.828 & 7.703 & $\mathrm{TL}$ \\
\hline Shapes4 & 8.485 & 32.675 & $\mathrm{TL}$ & 9.451 & 23.017 & $\mathrm{TL}$ & 8.485 & 31.276 & $\mathrm{TL}$ \\
\hline Poly1A [1-5] & 0.000 & 18.736 & $\mathrm{TL}$ & 7.120 & 18.592 & $12,435^{\mathrm{a}}$ & 0.000 & 18.720 & $\mathrm{TL}$ \\
\hline Poly1A [6-10] & 0.000 & 14.637 & $\mathrm{TL}$ & 6.027 & 14.518 & $12,161^{\mathrm{a}}$ & 0.000 & 15.889 & $\mathrm{TL}$ \\
\hline Poly1A [11-15] & 0.000 & 30.875 & $\mathrm{TL}$ & 4.600 & 21.661 & $13,680^{\mathrm{a}}$ & 0.000 & 27.616 & $\mathrm{TL}$ \\
\hline
\end{tabular}

TL, time limit was reached $(25,200.00 \mathrm{~s})$

a Out of memory

${ }^{\mathrm{b}}$ The optimality was proved

Considering $T R P M_{E}^{S B C}$ and $T R P M_{L}^{S B C}$, the SCIP solver found the best upper bounds for all instances, except for Poly1A[1-5], were the COUENNE solver found the best upper bound. Figure 10 presents the best solution found for each instance tested in this section. 
Table 6 Results found solving instances using $T R P M_{L}^{S B C}$ model

\begin{tabular}{|c|c|c|c|c|c|c|c|c|c|}
\hline \multirow[t]{2}{*}{ Instance } & \multicolumn{3}{|c|}{ COUENNE } & \multicolumn{3}{|l|}{ SCIP } & \multicolumn{3}{|c|}{ BARON } \\
\hline & LB & UB & TIME & LB & UB & TIME & LB & UB & TIME \\
\hline Three & 5.495 & 5.495 & $357^{\mathrm{b}}$ & 5.495 & 5.495 & $188^{\mathrm{b}}$ & 5.495 & 5.495 & $440^{\mathrm{b}}$ \\
\hline Blaze2 [1] & 2.828 & - & $\mathrm{TL}$ & 4.000 & 6.960 & $15,120^{\mathrm{a}}$ & 2.828 & 7.703 & $\mathrm{TL}$ \\
\hline Shapes4 & 8.485 & 35.481 & $\mathrm{TL}$ & 9.649 & 27.416 & $12,003^{\mathrm{a}}$ & 8.485 & 31.276 & $\mathrm{TL}$ \\
\hline Poly1A [1-5] & 0.000 & 18.395 & $\mathrm{TL}$ & 6.357 & 19.090 & $11,167^{\mathrm{a}}$ & 0.000 & 18.741 & $\mathrm{TL}$ \\
\hline Poly1A [6-10] & 0.000 & 15.462 & $\mathrm{TL}$ & 4.306 & 15.540 & $12,578^{\mathrm{a}}$ & 0.000 & 20.051 & $\mathrm{TL}$ \\
\hline Poly1A [11-15] & 0.000 & - & $\mathrm{TL}$ & 5.375 & 23.380 & $15,337^{\mathrm{a}}$ & 0.000 & 27.616 & $\mathrm{TL}$ \\
\hline
\end{tabular}

TL, time limit was reached $(25,200.00 \mathrm{~s})$

${ }^{\mathrm{a}}$ Out of memory

${ }^{\mathrm{b}}$ The optimality was proved

To better analyze the behavior of the model, the shapes 4 instance were run in SCIP iteratively using the $T R P M_{E}^{S B C}$ formulation. This solver and formulation were chosen because they presented the best performance by solving the instance. The model was solved three times and after each run, the solution obtained by the solver was used as a starting point for the next run. This process was repeated until the solver stopped to improve this solution, making a total of three runs. Figure 11 illustrates the solution obtained in the end of this process.

Comparing the solution in Fig. 11 with Shapes4 in Fig. 10, it can be observed that the solution had a significant improvement as the length reduced from 23.02 to 19.92. This behavior of the solution was expected because the starting point for the next run is always a better solution and the solver uses this solution to guide the search.

\section{Conclusions}

In this paper, a mixed integer quadratically-constrained programming model was proposed to represent the two-dimensional irregular strip packing problem considering that the pieces can be rotated in continuous angles. The model is flexible and can handle with polygonal convex and non-convex pieces. A set of symmetry breaking constraints were proposed and two variants of the model were analyzed, differing in the way that the non-overlapping constraints are addressed.

The mathematical formulation was solved using exact methods available in free solvers for academics. Computational experiments were performed using instances from the literature considering convex and non-convex pieces and showed that the proposed method found numerically accurate solutions in competitive computational times.

It can be stated that the SCIP solver performed better in the tests provided better lower bounds. Furthermore, the formulations presented in this paper are able to handle non-convex polygonal pieces in the irregular strip packing problem with continuous rotations.

Concerning this problem, to the best of our knowledge, the proposed model is the first in the literature to solve it using direct trigonometry to deal with the non-overlap between non-convex pieces. This simplicity in the representation of pieces and the non-overlapping constraint formulation makes the formulation simpler, making it adaptable to other variants of the problem of cutting and packing irregular pieces, which can be considered as future work. 
Three

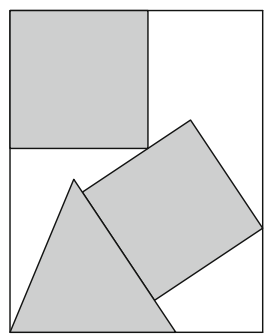

Length: 5.49
Blaze2[1]

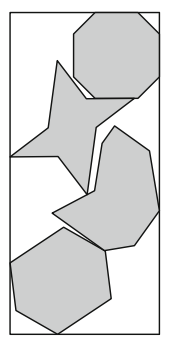

Length: 6.96
Shapes4

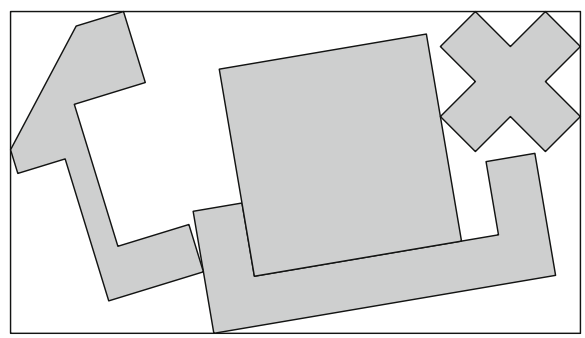

Length: 23.02
Poly1A[1-5]

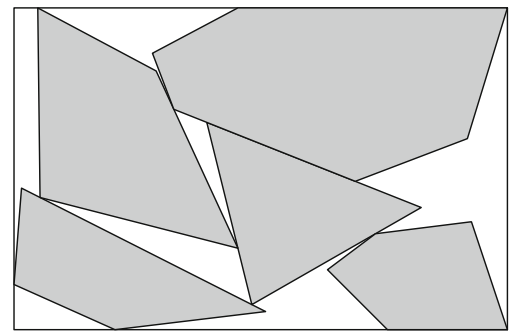

Length: 18.39
Poly1A[6-10]

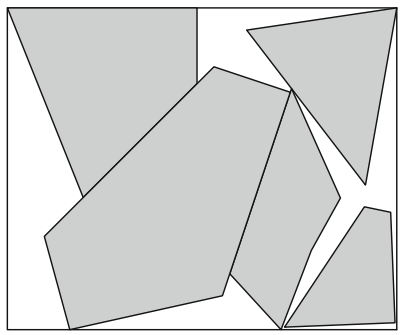

Length: 14.52

Poly1A[11-15]

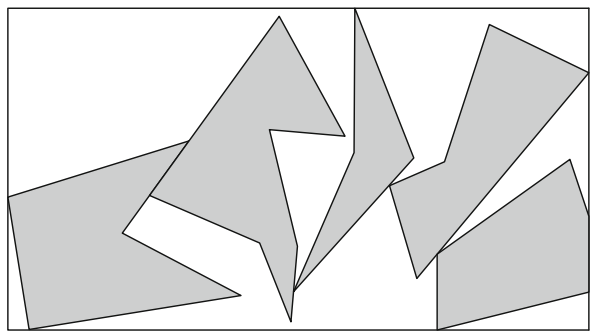

Length: 21.66

Fig. 10 The best solution found within $7 \mathrm{~h}$

Fig. 11 Solution of Shapes4 instance, found by SCIP in $T R P M_{E}^{S B C}$ after three runs of $7 \mathrm{~h}$.

The length of this solution is 19.92

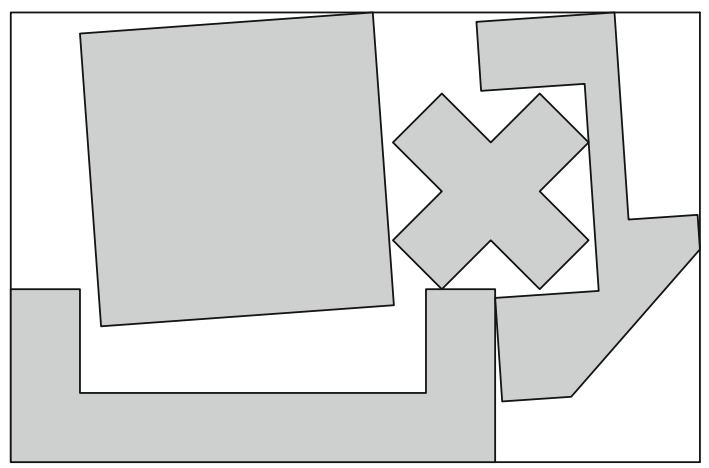


As the pieces are considered polygons, for many cases there is no approximation invoked in the geometry, therefore without time or space constraints the proposed models are exact.

Acknowledgements This research was sponsored and funded by FAPESP (2015/24987-4, 2015/03066-8 and 2013/07375-0) and CNPq (477481/2013-2).

\section{References}

1. Alvarez-Valdes, R., Martinez, A., Tamarit, J.: A branch \& bound algorithm for cutting and packing irregularly shaped pieces. Int. J. Prod. Econ. 145(2), 463-477 (2013)

2. Bennell, J., Scheithauer, G., Stoyan, Y., Romanova, T., Pankratov, A.: Optimal clustering of a pair of irregular objects. J. Glob. Optim. 61(3), 497-524 (2015)

3. Bennell, J.A., Oliveira, J.F.: The geometry of nesting problems: a tutorial. Eur. J. Oper. Res. 184(2), 397-415 (2008)

4. Bussieck, M.R., Meeraus, A.: General algebraic modeling system (GAMS). In: Kallrath, J. (ed.) Modeling Languages in Mathematical Optimization, Applied Optimization, vol. 88, pp. 137-157. Springer, New York (2004)

5. Carravilla, M.A., Ribeiro, C., Oliveira, J.F.: Solving nesting problems with non-convex polygons by constraint logic programming. Int. Trans. Oper. Res. 10, 651-663 (2003)

6. Chernov, N., Stoyan, Y., Romanova, T.: Mathematical model and efficient algorithms for object packing problem. Comput. Geom. 43(5), 535-553 (2010)

7. Cherri, L.H., Mundim, L.R., Andretta, M., Toledo, F.M., Oliveira, J.F., Carravilla, M.A.: Robust mixedinteger linear programming models for the irregular strip packing problem. Eur. J. Oper. Res. 253(3), 570-583 (2016)

8. Elkeran, A.: A new approach for sheet nesting problem using guided cuckoo search and pairwise clustering. Eur. J. Oper. Res. 231(3), 757-769 (2013)

9. Fischetti, M., Luzzi, I.: Mixed-integer programming models for nesting problems. J. Heuristics 15(3), 201-226 (2009)

10. Fowler, R.J., Paterson, M., Tanimoto, S.L.: Optimal packing and covering in the plane are np-complete. Inf. Process. Lett. 12(3), 133-137 (1981)

11. Fujita, K., Akagi, S., Hirokawa, N.: Hybrid approach for optimal nesting using a genetic algorithm and a local minimization algorithm. In: Proceedings of the 19th Annual ASME Design Automation Conference, Part 1, pp. 477-484 (1993)

12. Gomes, A.M., Oliveira, J.F.: A 2-exchange heuristic for nesting problems. Eur. J. Oper. Res. 141(2), 359-370 (2002)

13. Gottschalk, S.: Collision queries using oriented bounding boxes. $\mathrm{PhD}$ thesis, The University of North Carolina at Chapel Hill (2000)

14. Greene, D.H.: The decomposition of polygons into convex parts. Comput. Geom. 1, 235-259 (1983)

15. Jones, D.R.: A fully general, exact algorithm for nesting irregular shapes. J. Glob. Optim. 59(2-3), 367404 (2014)

16. Kallrath, J.: Cutting circles and polygons from area-minimizing rectangles. J. Glob. Optim. 43(2), 299-328 (2009)

17. Leão, A.A.S., Toledo, F.M.B., Oliveira, J.F., Carravilla, M.A.: A semi-continuous MIP model for the irregular strip packing problem. Int. J. Prod. Res. 54, 1-10 (2015)

18. Liao, X., Ma, J., Ou, C., Long, F., Liu, X.: Visual nesting system for irregular cutting-stock problem based on rubber band packing algorithm. Adv. Mech. Eng. 8(6), 1-15 (2016)

19. Rocha, P., Rodrigues, R., Toledo, F.M.B., Gomes, A.M.: Circle covering using medial axis. IFAC Proc. Vol. 46(7), 402-407 (2013)

20. Rocha, P., Rodrigues, R., Gomes, A.M., Toledo, F.M.B., Andretta, M.: Two-phase approach to the nesting problem with continuous rotations. IFAC PapersOnLine 48(3), 501-506 (2015)

21. Stoyan, Y., Pankratov, A., Romanova, T.: Cutting and packing problems for irregular objects with continuous rotations: mathematical modelling and non-linear optimization. J. Oper. Res. Soc. 67(5), 786-800 (2016)

22. Toledo, F.M.B., Carravilla, M.A., Ribeiro, C., Oliveira, J.F., Gomes, A.M.: The dotted-board model: a new mip model for nesting irregular shapes. Int. J. Prod. Econ. 145(2), 478-487 (2013) 